\title{
Strongyloides hyperinfection in a case of post- cadaveric solid organ transplantation
}

\author{
Nasir M Akhtar ${ }^{1}$, Kimberly J Fairley ${ }^{2}$, Andrew D Mowery ${ }^{2}$, Kimberly J Chaput ${ }^{2}$, Anil Kotru ${ }^{3}$, \\ Shaobo Zhu ${ }^{4}$, David L Diehl ${ }^{2}$, Michael J Komar ${ }^{2}$ \\ 1. Internal Medicine, Geisinger Medical Center, Danville, PA, USA. 2. Gastroenterology, Geisinger Medical Center, Danville, \\ PA, USA. 3. Transplant Surgery, Geisinger Medical Center, Danville, PA, USA. 4. Pathology, Geisinger Medical Center, \\ Danville, PA, USA.
}

Correspondence: Kimberly J Fairley. Address: Gastroenterology, Geisinger Medical Center, Danville, PA, USA. Email: kfairley2011@hotmail.com

Received: April 29, 2014

DOI : $10.5430 /$ crim.v1n2p242
Accepted: July 9, 2014

Online Published: August 25, 2014

URL: http://dx.doi.org/10.5430/crim.v1n2p242

\section{Abstract}

With cadaveric solid organ transplants becoming more common, the number of cases of infections transmitted from the donor to the recipient is increasing. This raises new questions regarding screening for infections in donors, particularly less common or rare infections.

A Caucasian, CMV-positive 64-year-old male with end-stage renal disease secondary to type I diabetes mellitus received a simultaneous left kidney/pancreas cadaveric transplant from a 24-year-old Hispanic, CMV-positive, HTLV-1 negative donor. The donor blood work was negative for eosinophilia, but he did receive steroids prior to organ procurement. Small bowel obstruction and gastrointestinal bleeding complicated the recipient's post-operative course. The patient had persistent nausea, bilious emesis, anorexia, and weight loss for months after his transplant. Endoscopy revealed confluent ulceration throughout the second portion of the duodenum with a heavy burden of the adult Strongyloides stercoralis on microscopic examination, consistent with hyperinfection. Urine and stool studies also confirmed the presence of Strongyloides infection. The patient was treated with ivermectin and immunosuppression changed to cyclosporine with resolution of the infection. Asymptomatic carriage was isolated to the donor (IgG positive status), while the archived recipient pre-transplant serum was negative for Strongyloides IgG. The other organ (liver, heart, and kidney) recipients were identified. The liver recipient died secondary to cardiopulmonary arrest post operative day 4 . The heart recipient developed episodes of organ rejection, was treated with steroids, and subsequently developed Strongyloides hyperinfection with pulmonary involvement. The recipient of the right kidney was asymptomatic.

This case highlights the need for enhanced screening protocols for cadaveric solid organ donors and recipients, based on infections endemic to the region of interest. In this circumstance, the identification of Strongyloides carriage may have reduced morbidity and mortality in the organ recipients.

\section{Keywords}

Strongyloides, Hyperinfection, Endoscopy, Transplantation

\section{I ntroduction}

Cadaveric solid organ transplant donors are screened for common infections such as hepatitis, cytomegalovirus (CMV), and human immunodeficiency virus (HIV). Yet, there are accounts of morbidity and mortality secondary to less common 
yet potentially preventable infections in the recipients ${ }^{[1]}$. We report a case of a middle-aged male who received cadaveric solid organ transplantation of a kidney and pancreas, and subsequently developed an acute strongyloidiasis hyperinfection.

\section{Case presentation}

A 64-year-old Caucasian male with history of CMV, coronary artery disease, and end-stage renal disease secondary to type I diabetes mellitus of 39 years duration received a simultaneous left kidney and pancreas cadaveric transplant in July 2012. The recipient often traveled on his motorcycle with his wife. He had recently traveled through Charleston, West Virginia to Tennessee. He frequently visited the southeastern United States and had no encounters with sick contacts within a month prior to transplantation. He denied overseas travel in the past. His archived pre-transplant serum was negative for S. stercoralis IgG. The donor was a 24-year-old Hispanic male born in Puerto Rico, but lived the majority of his life in Florida. He often visited Puerto Rico and was identified as healthy by family. He was admitted for gunshot wounds and his nine day hospital course resulted in a diagnosis of brain death. The donor's blood work was negative for eosinophilia, but he received high-dose steroids prior to organ procurement. He was identified as a CMV-positive, human T-lymphotropic virus-type I (HTLV-1) negative donor. The donor's kidneys, heart, and liver were transplanted into four recipients.

The recipient's post-operative course was complicated by small bowel obstruction, septic shock secondary to Enterobacter aerogenes bacteremia, and a pseudoaneurysm at the pancreatic-distal ileum anastomosis. These complications lead to allograft pancreatectomy, right hemicolectomy, and distal small bowel resection. Allograft pancreas evaluation demonstrated acute and chronic inflammation, fat necrosis, and focal hemorrhage. Distal ileum and colon biopsies revealed mucosal erosions and serositis. There was no evidence of S. stercoralis on biopsies. Intraoperative wound cultures were positive for Pseudomonas aeruginosa.

The patient presented to Gastroenterology two months after surgery with persistent nausea, bilious emesis, anorexia, and weight loss. On upper endoscopy, severe confluent ulceration (see Figure 1 A, B) and white villi (see Figure 2) were appreciated in the entire visualized duodenum. Endoscopic biopsies (see Figure 3 A, B, C) were positive for a heavy burden of the adult forms of Strongyloides, consistent with hyperinfection. A $5 \mathrm{~cm}$ by $5 \mathrm{~cm}$ fluid collection that caused extrinsic compression of the duodenum was appreciated on endoscopic ultrasound (EUS) and computed tomography (CT) scan of the abdomen (see Figure 4 A and B, respectively). An EUS guided fine needle aspiration was performed for diagnostic and therapeutic intent. Fluid cultures were negative for infection and repeat imaging demonstrated a decrease in the collection size. Stool and urine studies were also positive for Strongyloides. Ivermectin $15 \mathrm{mg}(0.2 \mathrm{mg} / \mathrm{kg})$ daily and albendazole $400 \mathrm{mg}$ twice daily were initiated for treatment duration of 6 weeks, followed by pulse dosing of the ivermectin. Mycophenolate mofetil 1000mg twice daily, prednisone 20mg daily, and tacrolimus 2mg daily were discontinued. Cyclosporine 75mg twice daily was initiated for immunosuppression and anti-helminthic properties. Repeat cultures and duodenal biopsies have been negative for persistent infection, and the patient is recovering well. Archived serum of donor and recipient from prior to transplantation was tested; Strongyloides infection was isolated to the donor (IgG positive status) supporting the notion that this was not a reactivated infection in the recipient.

A 14-year-old male with a single dysplastic kidney was the recipient of the right kidney at our institution. This individual had no travel history outside of the region or the United States. He was contacted after the renal/pancreatic transplant recipient was diagnosed. This patient was diagnosed with Strongyloides via stool testing and endoscopic biopsy. The patient was started on ivermectin and albendazole.

A 59-year-old Hispanic male with a history of ischemic cardiomyopathy was the recipient of the heart transplant. He was born in the United States but lived in Puerto Rico for 6 months as a teenager. The patient had episodes of organ rejection and was treated via steroids. He was admitted for a respiratory illness and received antibiotic, antiviral, and antifungal treatments. He underwent a bronchoscopy and was found to have $S$. stercoralis larvae. 
A 66-year-old Hispanic male with history of hepatitis $\mathrm{C}$ induced cirrhosis was the recipient of the liver transplant. The patient tolerated the initial transplant surgery. He became unresponsive on post-operative day 4 due to cardiac arrest and was unable to be resuscitated. Post mortem analysis was negative for S. stercoralis infection and definitive etiology for death was not appreciated.
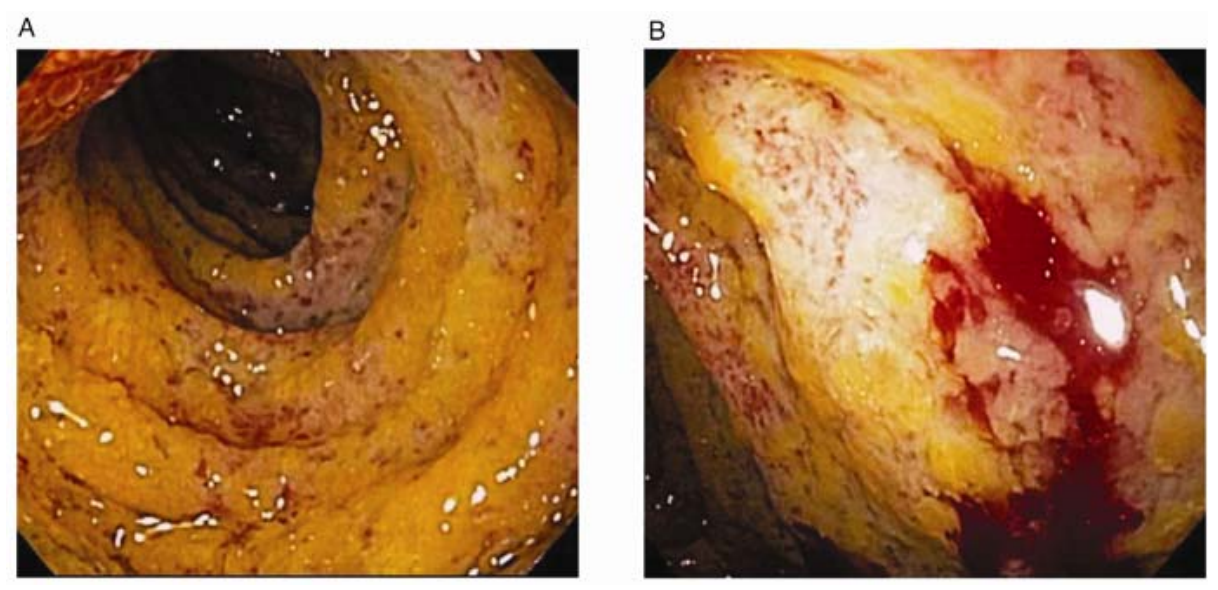

Figure 1 A B. Edematous and diffusely ulcerated duodenal mucosa.

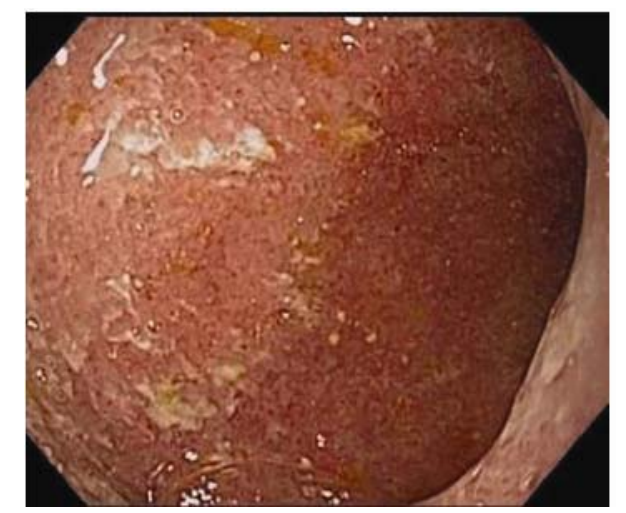

Figure 2. White villi seen in the duodenal bulb.
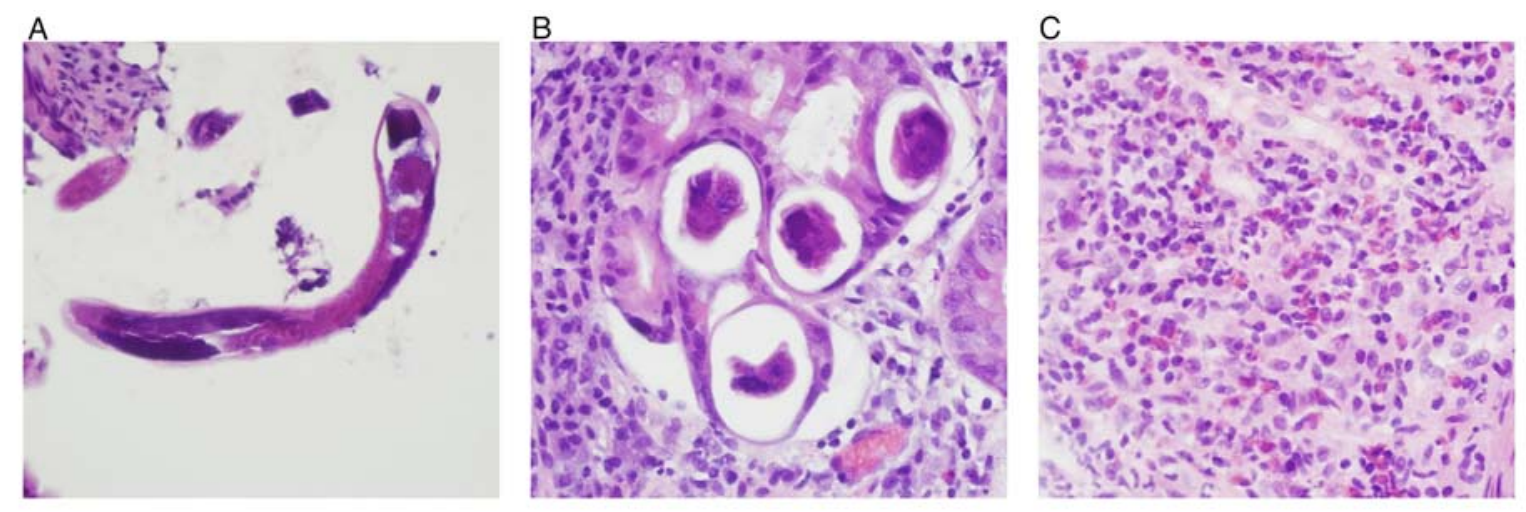

Figure 3. A. S. stercoralis infection in the duodenum. Longitudinal section of an adult worm, B. cross-section of $S$. stercoralis present in the lamina propria and crypts of duodenum, and C. eosinophilic infiltration in the duodenal mucosa (original magnification $\times 400$ in all). 

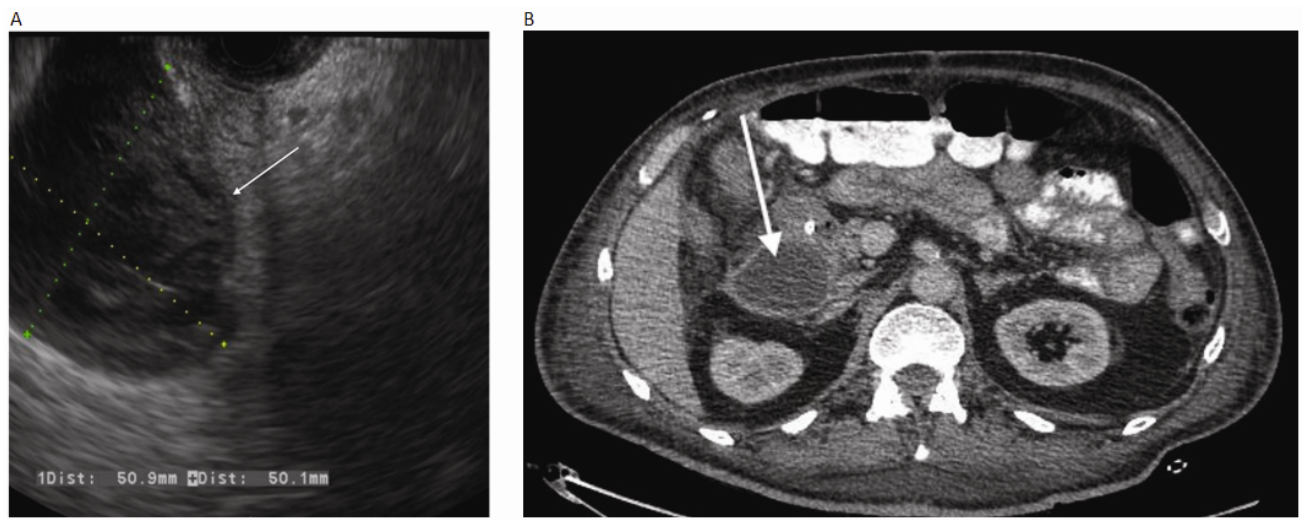

Figure 4. A. Fluid collection on EUS, and B. contrasted CT abdomen, indicated by arrows.

\section{Discussion}

Strongyloides stercoralis (S. stercoralis) is an intestinal nematode that is a human parasite that affects approximately 100 million people ${ }^{[2,3]}$. It is endemic in the subtropical and tropical regions such as the Caribbean Islands; but is also found in Africa, Asia, and the United States ${ }^{[4,5]}$. In the United States, endemic regions include Maryland, Kentucky, the Carolinas, eastern Tennessee, and West Virginia ${ }^{[4,6-8]}$. The city of Charleston, West Virginia reported 20 cases of $S$. stercoralis over a two year period, with an average age of 73.6 years and seventy percent of cases involving men ${ }^{[9]}$. Historically, the southeastern United States has also been an endemic region due to increased poverty and lack of plumbing infrastructure $^{[10]}$.

Almost all deaths in the United States from helminthes are due to S. stercoralis ${ }^{[6]}$. Strongyloides exists in parasitic and free-living stages. The filariform larvae are in the soil and penetrate skin as an individual travels barefoot, leaving larvae currens, a red cutaneous eruption of the skin ${ }^{[2,7]}$. The larvae travel via bloodstream to the lungs, migrate up the respiratory tract, are swallowed, and enter the gastrointestinal tract to ultimately reside in the duodenum. In the duodenum, the larvae mature into female worms that lay up to 50 eggs per day ${ }^{[6,7]}$. The eggs hatch and the larvae are either passed in the stool or penetrate the colon and perianal sphincter to renter circulation and move toward the lungs to continue the parasitic cycle ${ }^{[7]}$.

The clinical presentation of strongyloidiasis is non-specific with symptoms including abdominal pain, diarrhea, vomiting, ileus, bowel obstruction, or pneumonia. Chronic infection is asymptomatic in $50 \%$ of individuals, but $75 \%$ of individuals manifest eosinophilia ${ }^{[2,11]}$. Disseminated infection entails migration of larvae beyond the pulmonary and gastrointestinal tract; this is often complicated via gram-negative sepsis due to translocation of intestinal bacteria ${ }^{[4]}$. Mortality rate for disseminated infections are as high as $87 \%{ }^{[12]}$. Hyperinfection is accelerated autoinfection in the immunosuppressed, noted by an increased amount of larvae released into venous circulation. There are also increased larvae in stool, sputum, and/or tissue ${ }^{[2]}$. Although less than $3 \%$ of cases of S. stercoralis infections involve hyperinfection or disseminated infection ${ }^{[2]}$, infected patients are often immunosuppressive agents such as corticosteroids ${ }^{[7]}$. Complicated presentations of hyperinfection syndrome include hematemesis due to hemorrhagic duodenitis, partial small bowel obstruction, or Loffler's syndrome ${ }^{[11]}$. Given the complications, an early diagnosis is important as strongyloidiasis is a readily treatable infection ${ }^{[2]}$. In post-transplantation patients, ileus or small bowel obstruction are the presenting symptom in $21 \%$ of cases, and gastrointestinal hemorrhage in $15 \%$ of cases ${ }^{[13]}$. Our patient had both, which was likely the first sign of his hyperinfection state.

Traditionally, the diagnosis of strongyloidiasis is difficult as it requires the observation of larva ${ }^{[3]}$. Diagnosis is often made via stool examination; however a single stool examination is unable to detect larvae in up to $70 \%$ of cases ${ }^{[2,7]}$. Studies have shown that 7 serial stool examinations lead to a sensitivity of $99 \%{ }^{[2]}$. This method also requires fresh stool to be 
collected and is a health risk for individuals handling the stool ${ }^{[3]}$. It should be noted that negative stool studies do not exclude Strongyloides as the intestinal worm load may be low and larvae output might be minimal. Various immunological methods have been developed to improve detection of Strongyloides infection; however variation exists in sensitivity and specificity between methods. Individuals with strongyloidiasis infection develop antibodies in response; IgG can be detected two weeks post-infection, with peak at 6 weeks, and up to 20 weeks after infection ${ }^{\text {[14] }}$. The organ donor's archived pre-transplantation serum was IgG positive. IgM antibody is present in acute infections, peaking at 1 week duration and lasting approximately 3 weeks. In individuals with hyperinfection, there is a blunt in response of IgM but not IgG ${ }^{[14]}$.

Enzyme-linked immunosorbent assay (ELISA) for Strongyloides IgG antibodies is commonly available and has increased practicality - allowing detection of larvae either in stool or via serum ${ }^{[14]}$. It has a sensitivity of $83 \%-93 \%$ and specificity of $95 \%-97.7 \%$ in determining if an infection has occurred at any time ${ }^{[4,15]}$. There is significant association between positive serology and present infection, and thus stool studies should be obtained for further evaluation as cross-reactivity may occur with other helminthes such as filaris ${ }^{[14]}$. Recent studies suggest improved methods for diagnosis via use of 31-kDa recombinant antigen (NIE), which has no interaction with other helminthes. Use of NIE-based assays in conjunction with Luciferase Immunoprecipitation System (NIE-LIPS) approaches $97 \%$ sensitivity and $100 \%$ specificity for diagnosis of $S$. stercoralis ${ }^{[3,16]}$. In our case, endoscopy provided the diagnosis as Strongyloides was colonized in the duodenum. Generalized endoscopic findings for strongyloidiasis range from friable edema to ulceration to necrotic mucosa to mucosal granularity; however none of these features are specific for strongyloidiasis ${ }^{[7,13,17]}$. Our patient had severe ulcerations and white villi; the latter demonstrates dilated lymphatics and impaired transport of fats from the epithelium to the intestinal lymphatic system. Strongyloides disrupts the epithelium of the duodenum via apoptosis and halting cellular proliferation leading to villous atrophy and decreased absorption ${ }^{[2]}$.

Healthy individuals are typically asymptomatic, even in setting of chronic infection. Risk factors for disseminated disease and hyperinfection include hematologic malignancy, alcoholism, immunosuppressive therapy (mostly prednisone), kidney transplantation, diabetes, and HTLV-1 ${ }^{[6,11]}$. Our patient received prednisone, methylprednisolone, and underwent organ transplantation. Corticosteroids reduce proliferation of eosinophils and monocytes; along with induction of apoptosis in eosinophils and immature lymphocytes ${ }^{[11]}$. Steroids are also linked to an increase output of infectious larvae ${ }^{[2,11]}$. It is less common for individuals to have eosinophilia in a state of hyperinfection, with the association conferring an improved prognosis ${ }^{[18]}$. Cadaveric transplantation has become more common as demonstrated with our patient who received two organs. Renal transplants have been associated with strongyloidiasis hyperinfection in $0.7 \%$ of cases ${ }^{[13]}$. It is postulated that kidneys may transmit $S$. stercoralis from donor to recipient as larvae have been found in the urine of both renal transplanted and non-transplanted individuals ${ }^{[18]}$.

Of all immunosuppressive agents prescribed, glucocorticoids are most associated with the transition from chronic strongyloidiasis to hyperinfection. Signs and symptoms of infection begin as early as 20 days after treatment and as late as years after use ${ }^{[18]}$. It is suggested that glucocorticoids may accelerate the transformation of larvae from latent form to invasive form ${ }^{[18]}$. On the other hand, there is a lack of association between cyclosporine and S. stercoralis hyperinfection. In the 1990s, it was noted that S. stercoralis infections decreased upon renal transplant patients after the introduction of cyclosporine. It is hypothesized that cyclosporine has an anti-helminthic effect ${ }^{[18]}$.

The challenge presented in our clinical scenario was to determine the risk of both donor and recipient having an opportunistic infection such as strongyloidiasis. Various constraints potentially prohibited investigation for S. stercoralis pre-transplantation. Living donor screening involves a history and physical, serologic and laboratory testing, and potential radiographic studies. Commonly tested diseases include HIV, HTLV, herpes simplex virus, hepatitis B, hepatitis C, Epstein Barr virus, and varicella zoster virus ${ }^{[19]}$. Starting February 2013, the Organ Procurement and Transplantation Network (OPTN) mandated testing for Strongyloides for donors from endemic regions ${ }^{[20]}$. Deceased donor organ evaluation is often done in hours with laboratory and serologic testing potentially limited to HIV and hepatitis C. Patient 
history is heavily weighed to determine pathogen screening. Most solid organ transplant centers do not routinely screen for Strongyloides, with survey showing that $9 \%$ of US-trained physicians recognize cases needing screening for Strongyloides ${ }^{[4,5]}$. Strongyloides screening may have been overlooked as the donor was identified as a healthy, was without eosinophilia, and due to time constraints of deceased organ donor evaluation. Neither donor nor recipient had eosinophilia due to steroids therapy ${ }^{[4,5]}$. Steroids were administered to increase organ viability and donor stability for organ procurement ${ }^{[20,21]}$. Conversely, steroid use has been recognized for triggering reactivation and potential dissemination of a previously latent infection to the kidneys in the previously noted healthy donor. Donor screening strategies are challenging due to exposure, history, and limited time for evaluation ${ }^{[21]}$.

The donor was of Hispanic heritage. A survey conducted in other countries show S. stercoralis is prevalent in Latin America. In Brazil, the prevalence is between 15\% and $82 \%$; in Costa Rica, S. stercoralis is found between $1.1 \%$ and $16.5 \%{ }^{[22]}$. The Caribbean is considered an endemic region as numerous Jamaicans and Cubans living in the United States have been reported in having disseminated hyperinfection ${ }^{[22]}$. Although the donor was neither Jamaican nor Cuban, he visited Puerto Rico, an endemic region ${ }^{[23]}$. In neighboring Canada, 77.5\% of immigrants between 1991 and 2001 were from Strongyloides epidemic countries; this leads to the question of the number of individuals immigrating in the United States who have Strongyloides ${ }^{[24]}$. In 1996, the United States had approximately 600,000 immigrants from countries that were endemic to $S$. stercoralis ${ }^{[25]}$. Given cost and low-side effect profile of albendazole, if all 600,000 immigrants were prophylactically treated, 374 hospitalizations would have been saved along with 33 lives ${ }^{[25]}$.

Strongyloidiasis should be in the differential for cadaveric solid organ transplantation, both in asymptomatic donors and in selected recipients. Risk factors for asymptomatic carriage include recent use of steroids, known HTLV-1 infection, unexplained eosinophilia, remote travel history or residences in epidemic regions, or prior military service ${ }^{[18,20]}$. Primary prevention with ivermectin or albendazole can be considered in patients who have positive serologic studies and/or history of exposure but do not have hyperinfection ${ }^{[18]}$. For those who have strongyloidiasis, ivermectin is the treatment of choice, although there is paucity of randomized trials dictating treatment protocols ${ }^{[7,26]}$. This case highlights the current knowledge gap and need for enhanced screening protocols for cadaveric solid organ donors and recipients, based on infections endemic to the region of interest even in areas with low prevalence. In this circumstance, the identification of Strongyloides asymptomatic carriage in the donor (who had risk factors for this infection) may have reduced morbidity and mortality in the organ recipients.

\section{Conclusion}

Strongyloidiasis should be in the differential for patients on immunosuppression or history of cadaveric solid organ transplantation that present with gastrointestinal symptoms. The recipient had risk factors leading to his hyperinfection: the donor having Strongyloides, steroid therapy, and renal transplantation ${ }^{[15]}$. Individuals who receive steroids, have HTLV-1, unexplained eosinophilia, remote travel history or residences in epidemic regions, or prior military service should be screened for Strongyloides ${ }^{[18,20]}$. Primary prevention with ivermectin or albendazole can be considered in patients who have positive serologic studies and/or history of exposure but do not have hyperinfection ${ }^{[18]}$. For those who have strongyloidiasis, ivermectin is the treatment of choice, such that a single dose has a cure rate of $85 \%$ to $94 \%{ }^{[7,26]}$. The cure rate is $100 \%$ upon administration of two consecutive daily courses ${ }^{[7]}$. This case highlights the current knowledge gap and need for enhanced screening protocols for cadaveric solid organ donors and recipients, based on infections endemic to the region of interest even in areas with low prevalence. In this circumstance, the identification of Strongyloides carriage may have reduced morbidity and mortality in all of the organ recipients from the infected donor.

\section{References}

[1] Delmonico, FL. Cadaver donor screening of infectious agents in solid organ transplantation. Clin Infect Dis. 2000; 31:781-86. http://dx.doi.org/10.1086/314000

Published by Sciedu Press 
[2] Kishimoto K, Hokama A, Hirata T, Ihama Y, Nakamoto M, Kinjo N, Kinjo F, Fujita J. Strongyloides pic and histopathological study on the duodenum of Strongyloides stercoralis hyperinfection. World J Gastroentero. 2008; 14(11): 1768-73. http://dx.doi.org/10.3748/wjg.14.1768

[3] Anderson NW, Klein DM, Dornink SM, Jespersen DJ, et al. Comparison of three immunoassays for detection of antibodies to Strongyloides stercoralis. Clin Vaccine Immunol. 2014; 21: 732-6. http://dx.doi.org/10.1128/CVI.00041-14

[4] Roxby AC, Gottlieb GS, Limaye AP. Strongyloidiasis in Transplant Patients. Clin Infect Dis. 2009; 49: 1411-23. http://dx.doi.org/10.1086/630201

[5] Weiser JA, Scully BE, Bulman WA, Husain S, Grossman ME. Periumbilical parasitic thumbprint purpura: strongyloides hyperinfection syndrome acquired from a cadaveric renal transplant. Transpl Infect Dis. 2011; 13: 58-62. http://dx.doi.org/10.1111/j.1399-3062.2010.00516.x

[6] Kakati B, Dang S, Heif M, Caradine K, McKnight W, Aduli F. Strongyloides duodenitis: case report and review of literature. J Natl Med Assoc. 2011; 13(1): 60-63

[7] Overstreet K, Chen J, Rodriquez JW, Wiener G. Endoscopic and histopathologic findings of strongyloides stercoralis infection in a patient with AIDS. Gastrointest Endoscopy. 2003; 58(6): 928-31. http://dx.doi.org/10.1016/S0016-5107(03)02280-6

[8] Berk SL, Verghese A, Alvarez S, Hall K, Smith B. Clinical and epidemiologic features of strongyloidiasis. A prospective study in rural Tennessee. Arch Intern Med. 1987; 147: 1257-61. http://dx.doi.org/10.1001/archinte.1987.00370070071011

[9] Kitchen LW, Tu KK, Kerns FT. Strongyloides-infected patients at Charleston area medical center, West Virginia, 1997-1998. Clin Infect Dis. 2000; 31: E5-6. http://dx.doi.org/10.1086/314022

[10] Starr MC, Montgomery SP. Soil-transmitted helminthiasis in the United States: a systematic review-1940-2010. Am J Trop Med Hyg. 2011; 85: 680-4. http://dx.doi.org/10.4269/ajtmh.2011.11-0214

[11] Concha R, Harrington W, Rogers AI. Intestinal strongyloidiasis recognition, management, and determinants of outcome. J Clin Gastroenterol. 2005; 39(3): 203-11. http://dx.doi.org/10.1097/01.mcg.0000152779.68900.33

[12] Olsen A, van Lieshout L, Marti H, Polderman T, Polman K, Steinmann P, et al. Strongyloidiasis--the most neglected of the neglected tropical diseases? Trans R Soc Trop Med Hyg. 2009; 103: 967-72. http://dx.doi.org/10.1016/j.trstmh.2009.02.013

[13] Hiwaza K, Iida M, Aoyagi K, Kimura Y, Eguchi K, Fujishima M. Early detection of strongyloidiasis using endoscopy duodenal biopsy: report of a case. J Clin Gastroenterol. 1996; 22(2): 157-59. http://dx.doi.org/10.1097/00004836-199603000-00021

[14] Levenhagen MA, Costa-Cruz JM. Update on immunologic and molecular diagnosis of human strongyloidiasis. Acta Trop. 2014; 135: 33-43. http://dx.doi.org/10.1016/j.actatropica.2014.03.015

[15] Marcos LA, Terashima A, Dupont HL, Gotuzzo E. Strongyloides hyperinfection syndrome: an emerging global infectious disease. Trans R Soc Trop Med Hyg. 2008; 102: 314-8. http://dx.doi.org/10.1016/j.trstmh.2008.01.020

[16] Bisoffi Z, Buonfrate D, Sequi M, Mejia R, Cimino RO, et al. Diagnostic accuracy of five serologic tests for Strongyloides stercoralis infection. PLoS Negl Trop Dis. 2014; 8: e2640. http://dx.doi.org/10.1371/journal.pntd.0002640

[17] Thompson FB, Fry LC, Wells CD, Olmos M, Lee DH, Lazenby AJ, Monkemuller KE. The spectrum of GI strongyloidiasis: an endoscopic-pathologic study. Gastrointest Endoscopy. 2004; 59(7): 906-10. http://dx.doi.org/10.1016/S0016-5107(04)00337-2

[18] Keiser PB, Nutman TB. Strongyloides stercoralis in the immunocompromised population. Clin Microbiol Rev. 2004 ; $17: 208-17$. http://dx.doi.org/10.1128/CMR.17.1.208-217.2004

[19] Fischer, SA, Avery RK, and the AST Infectious Disease Community of Practice. Screening of Donor and Recipient Prior to Solid Organ Transplantation. Am J Transplant. 2009; 9: S7-S18. http://dx.doi.org/10.1111/j.1600-6143.2009.02888.x

[20] Levi ME, Kumar D, Green M, Ison MG, Kaul D, et al. Considerations for screening live kidney donors for endemic infections: a viewpoint on the UNOS policy. Am J Transplant. 2014 May; 14(5): 1003-11. http://dx.doi.org/10.1111/ajt.12666

[21] Hamilton KW, Abt PL, Rosenbach MA, Bleicher MB, Levine MS, et al. Donor-derived Strongyloides stercoralis infections in renal transplant recipients. Transplantation. 2011; 9: 1019-24. http://dx.doi.org/10.1097/TP.0b013e3182115b7b

[22] Genta RM. Global prevalence of strongyloidiasis: critical review with epidemiologic insights into the prevention of disseminated disease. Rev Infect Dis. 1989; 11(5): 755-67. http://dx.doi.org/10.1093/clinids/11.5.755

[23] Huston JM, Eachempati SR, Rodney JR, et al. Treatment of Strongyloides stercoralis hyperinfection-associated septic shock and acute respiratory distress syndrome with drotrecogin alfa (activated) in a renal transplant recipient. Transpl Infect Dis. 2009; 11: 277-80. http://dx.doi.org/10.1111/j.1399-3062.2009.00386.x

[24] Lim S, Katz K, Krajden S, Fuksa M, Keystone J, Kain K. Complicated and fatal strongyloides infection in Canadians: risk factors, diagnosis and management. Can Med Assoc J. 2004; 171(5): 479-84. http://dx.doi.org/10.1503/cmaj.1031698

[25] Muennig P, Pallin D, Sell RL, Chan MS. The cost effectiveness of strategies for the treatment of intestinal parasites in immigrants. New Eng J Med. 1999; 340: 773-79. http://dx.doi.org/10.1056/NEJM199903113401006

[26] Siddiqui AA, Berk SL. Diagnosis of strongyloides stercoralis infection. Clin Infect Dis. 2001; 33(7): 1040-47. http://dx.doi.org/10.1086/322707 